\title{
Analysis of Low Liquid Loading Two-Phase Flow Using CFD and Experimental Data
}

\author{
Miguel Ángel Ballesteros Martínez ${ }^{1}$, Nicolás Ratkovich ${ }^{1}$, Eduardo Pereyra² \\ ${ }^{1}$ Department of Chemical Engineering, Universidad de los Andes \\ Carrera 1E \#19A-40, Bogota, Colombia \\ ma.ballesteros641@uniandes.edu.co; n.rios262@uniandes.edu.co \\ ${ }^{2}$ McDougall School of Petroleum Engineering, University of Tulsa \\ 800 S Tucker Dr, Tulsa, USA \\ eduardo-pereyra@utulsa.edu
}

\begin{abstract}
Low liquid loading flow occurs very commonly in the transport of any kind of wet gas, where the changing pressure and temperature conditions along the pipeline cause the condensation of hydrocarbon gases and water. The presence of even a small amount of liquid load in the fluid has significant effects on the flow conditions, such as an important increase in the pressure drop and the formation of annular or stratified flow patterns. It can cause important flow assurance issues as well, such as pipe corrosion or unexpected wall stress on the pipeline. This makes the modelling and analysis of these types of flow particularly important to the Oil \& Gas industry, in order to improve the design and operation of gas pipelines and downstream facilities. Because of that, this study focused on modelling this type of flow and analysing the effects of moderate or high pressures on the flow conditions, in medium diameter (6-in) pipes, using CFD simulation. A polyhedral mesh was used, and is fineness was determined with a mesh independence test. The physics models were tested against experimental data obtained at the University of Tulsa, focusing mainly on the turbulence and interface models. The selected turbulence model was the $\mathrm{k}-\omega$ model, and the analysis on the interface models is still ongoing. The Interface Momentum Dispersion model, however, has promising results, though it requires a high computational cost. An inverse relation between the pressure and the liquid holdup was still able to be determined, since the higher gas density and viscosity cause a higher drag force on the liquid face, which reduces its holdup.
\end{abstract}

Keywords: Low Liquid Level Flow, CFD, Liquid Holdup, Turbulence Models, Interface Models.

\section{Introduction}

Low liquid loading flow occurs very commonly in many industries, such as the Chemical, Nuclear, and Oil \& Gas Industries[1], [2], where it is usually referred to as wet gas. The exact definition of a wet gas flow is still a matter of debate. Meng [1] defines it as any two-phase flow in which the liquid volumetric flow rate is less than $1100 \mathrm{~m}^{3}$ per $\mathrm{MMsm}^{3}$ of gas volumetric flow rate. In the Oil \& Gas Industry, it is common to call wet gas any flow with a gas volume fraction greater than 90\%[3], [4]. However, since gas is a compressible fluid, it is important to account for the densities of the fluid phases, as well as the flow rate. Therefore, it has become common practice to define the wetness of a gas using the Lockhart Martinelli parameter. As for the limit value for this parameter, the Norwegian Society for Oil and Gas Measurement (NFOGM), the American Petroleum Institute (API) and the American Society of Mechanical Engineering (ASME) all state that a wet gas flow is any two-phase flow that has a value of less than, or equal to, 0.3 [3], [5].

In the Oil \& Gas Industry, wet gas tends to be a particularly common occurrence in the transport of natural gas from deep wells, where the changing conditions along the pipeline causes condensation of hydrocarbon gases and/or water vapour [6], [7]. Even if the natural gas enters the pipeline as a single-phase flow, as it ascends to downstream facilities, the decrease in pressure will cause it to turn into a two-phase flow, or even a multiphase one, if both hydrocarbons and water condensate.

Any amount of liquid can have important effects on the pipeline flow conditions and assurance, such as an increase in the pressure loss along the pipeline [8]. A trace amount of condensate of as little of $0.5 \%$ volume fraction in the gas flow may cause an increment of as much as $30 \%$ in the pressure gradient along the pipeline. Liquid holdup along the pipes must also be taken into account when determining pigging frequency and designing any downstream facility. It is important as 
well when analysing wax deposition and hydrate formation in the pipelines [1]. Wall stresses, which are significantly larger with the presence of a liquid phase, may have an effect on the selection of pipe material during pipeline design and affect corrosion [7]. Finally, the presence of liquid can cause errors of as much as $60 \%$ in the measurement of gas flow rate, which can cause serious issues in flow assurance. In order to correct the measuring equipment, not only is the liquid holdup needed, but also the flow pattern of the two-phase flow [3]. All of this makes the modelling and analysis of low liquid loading, or wet gas, flow very important for the industry.

The analysis of wet gas flows can be done through mostly two different types of studies: experimental and computational. However, despite the importance of low liquid loading flow study, there is only a limited amount of studies focused on this topic. Experimental studies so far have used only small-diameter horizontal pipes, medium pressures and mostly water-air mixtures[6], [8], [9]. Only Duc [10] and Dong [11] have performed studies on oil-air mixtures in mediumdiameter pipes (6 in). Computational studies are even more limited. They have focused mostly in low-pressure stratified, smooth or wavy, flows of water-air mixtures, and the models have not shown to predict properly flow conditions at low liquid holdups [7], [12].

\section{Materials and Methods}

Because of the reasons mentioned earlier, this study will focus on the computational analysis of the effect of pressure on the flow conditions of two-phase flows (gas and condensate) in a 0.15-m pipe, using STAR-CCM+ for Computational Fluid Dynamic (CFD) analysis. CFD simulations were chosen in order to avoid high experimental costs and to be able to simulate conditions beyond the reach of physical experimental facilities. First, a mesh independence test was performed, to ensure the spatial discretization and the meshing method did not affect the value flow conditions predicted by the simulations, using the data obtained Karami [7], on a $4.57 \mathrm{~m}$ pipe with a water - air mixture, as a point of reference. Then, different turbulence models were evaluated, to observe their effect on the liquid holdup predicted and on the computational time required. Finally, the physical models were compared with experimental results obtained by Duc [10], who used a larger experimental facility, with a pipe length of over $12.19 \mathrm{~m}$, with an Isopar L oil - nitrogen mixture. Three different operating pressures were used, to compare the effect it caused on the experimental and computational results. Based on this results, an analysis on the interface model was also performed.

\subsection{Mesh and Boundary Conditions}

Two different meshes were evaluated in order to simulate the low-liquid-level flow: orthogonal and polyhedral. The orthogonal grid is based on a rectangular pattern at the core of the pipe, and a finer cylindrical meshing around it. This allows refining the grid near the pipe wall, while maintaining a coarser mesh at the core, and prevents a singularity at the centre of the pipe [13], as it can be seen Fig. 1 (a). The other type is a polyhedral mesh, which has more uniform cells along the pipe line but requires a finer mesh, as it can be also seen in Fig. 1 (b). An independence test was done for both of these mesh types. In case of the polyhedral mesh, the cell base size was simply altered to obtain different cell numbers. For the orthogonal mesh, both the axial and the radial divisions were changed, so the effect of each of them could be analysed.

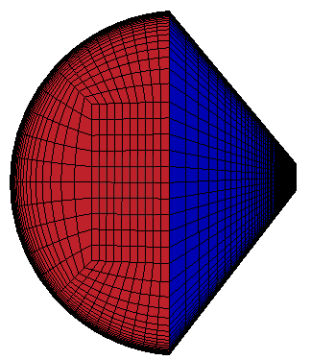

(a)

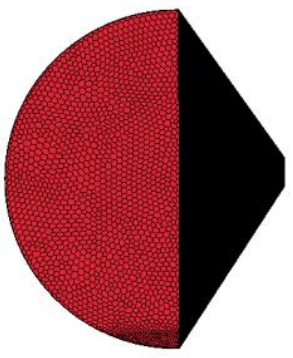

(b)

Fig. 1: Different types of meshes used: (a) orthogonal (b) polyhedral.

This analysis, as well as the turbulence and interface model tests, were performed using the geometry used by Karami [14], which was a $0.15-\mathrm{m}$ pipe, with a length of $4.57 \mathrm{~m}$. The superficial velocity of the gas was set to $10 \mathrm{~m} / \mathrm{s}$, and the liquid 
velocity, to $0.02 \mathrm{~m} / \mathrm{s}$. The operating pressure was set to $0.06 \mathrm{MPa}$, and the system was simulated for $18 \mathrm{~s}$. As for the experimental comparison, the geometry was based on Duc's [10] study, which used a 0.15-m pipe as well, but with a larger length, of which $12.2 \mathrm{~m}$ was simulated. The gas and liquid velocities were set to the same values as for the previous simulations. Three different operating pressures were simulated: 1.48, 2.17 and 2.86 MPa. The liquid holdup was selected as comparison criterion for all tests. Its selection was based on the fact that it is a particularly important parameter in flow assurance and that due to its low order of magnitude, numerical and mathematical model errors would have a more immediately evident effect on its value.

\subsection{Experimental Facility}

The experimental data used for the model validation was collected at the University of Tulsa, in TUFFP 0.15-m ID high-pressure two-phase flow facility, by Duc [10]. This facility is capable of conducting single-phase gas and two-phase gas-oil experiments, at system pressures of up to psig and inclinations of $-3^{\circ}$ to $3^{\circ}$. The oil pumping system uses Isopar $\mathrm{L}$ mineral oil, which it can deliver at a flow rate of as much as $3.9 \mathrm{~m}^{3} / \mathrm{h}$, using a Moyno Progressing Cavity pump. The gas compressing system delivers nitrogen at $21250 \mathrm{~m}^{3} / \mathrm{h}$, using a BMC-343 EF compressor. Flow rates are measured using two Micro Motion Coriolis mass flow meters: the CMF 100, which is for low flow rates, and the CMF 300, which is for high flow rates.

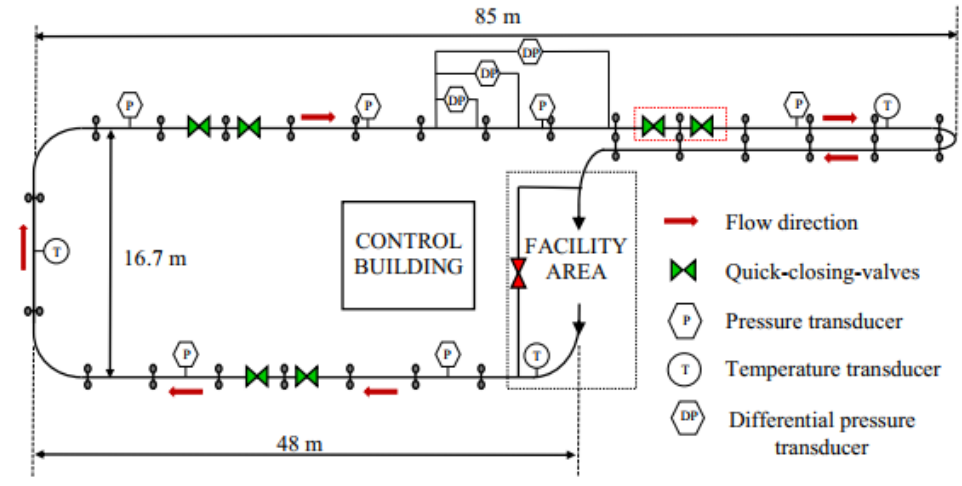

Fig. 2: Diagram of the TUFFP 0.15-m ID high-pressure two-phase flow facility at the University of Tulsa. Taken from Duc [10].

The test pipe section has a total length of $523 \mathrm{ft}(159.4 \mathrm{~m})$ and is divided in two straight sections. The longest straight section has a length of $279 \mathrm{ft}(85 \mathrm{~m})$, and it can be inclined up to $3^{\circ}$ upward from right to the left in the diagram shown in Fig. 2. The flow measurement instruments are installed in the middle of the fully developed section. For analysing flow and operating conditions, the facility is equipped with: temperature transmitters, pressure transmitters, differential pressure transducers, quick closing valves, a wired-mesh sensor, iso-kinetic probes and a Canty visualization system. Both the wired mesh sensor and the Canty visualization system are used to determine the liquid hold-up and to analyse the flow pattern. The wired mesh can provide an idea of the distribution of the liquid phase across the pipe cross-sectional area [15], while the Canty visualization system provides a better measurement of the liquid hold-up at low liquid loading conditions [10]. The iso-kinetic sampling system, on the other side, was used to measure the amount of the liquid phase that was entrained in the gas flow.

\subsection{Fluid Properties}

The fluids simulated for each different test depended on whose experimental data was used for comparison, whether Karami [7] or Duc [10]. For all cases, the temperature used was the average temperature of Tulsa, which is around $18^{\circ} \mathrm{C}$ [16]. For the mesh independence and the turbulence model selection tests, the experimental data from Karami was selected, who used an air - water mixture. Since the gauge pressure inside the pipe section did not surpass the $0.06 \mathrm{MPa}$, standard pressure conditions were assumed when calculating the fluid properties. In order to ease the computational cost, all properties were assumed constant for each simulation. The property values are shown in Table 1 . 
Table 1: Fluid properties used for the different simulations.

\begin{tabular}{|c|c|c|c|c|}
\hline & Air & Water & Nitrogen & Isopar L \\
\hline Viscosity $(\mathbf{P a} \cdot \mathbf{s})$ & $1.86 \times 10^{-5}$ & $8.89 \times 10^{-4}$ & $1.78 \times 10^{-5}$ a & 0.0013 \\
\hline Density $\left(\mathbf{k g} / \mathbf{m}^{\mathbf{3}}\right)$ & 2.05 & 997.56 & $16.02^{\mathrm{a}}$ & 760 \\
\hline Surface tension $(\mathbf{N} / \mathbf{m})$ & \multicolumn{2}{|c|}{0.074} & \multicolumn{2}{|c|}{0.024} \\
\hline
\end{tabular}

${ }^{\text {a }}$ The values shown for Nitrogen properties are an average of the values used in the different simulations for the experimental validation.

As it was mentioned before, the fluids used by Duc [10] were Isopar L oil and nitrogen. The properties of each fluid were calculated for each of the operating pressures simulated. Since Duc determined that the pressure and the temperature varied no more than $1 \mathrm{psig}$ and $1{ }^{\circ} \mathrm{C}$ across the test section, the properties were assumed as constant for each simulation. For the Isopar L oil, the properties used were the same for every simulation, as they are shown in Table 1. As for the nitrogen, its properties were calculated for each operating pressure. The density of the gas was predicted using Span et al. [17] correlation, which is shown in Eq. (1). For this correlation, $\rho$ is in $\mathrm{kg} / \mathrm{m} 3, \mathrm{P}$ is the pressure in $\mathrm{Pa}$, T is in $\mathrm{K}$, and $\mathrm{R}$ is the specific gas constant of nitrogen. The viscosity of nitrogen, on the other hand, was calculated using Seibt et al. [18] correlation, which is shown in Eq. (2).This correlation depends on the reduced variables $\delta$ and $\tau$, whose equations are shown in Eq.(3). For this correlation, $\mu$ is the viscosity in $\mu \mathrm{Pa} \cdot \mathrm{s}$. All other variables refer to parameters of the correlations.

$$
\begin{gathered}
\rho=\frac{P}{R T\left[1+\sum_{k=1}^{10} i_{k} N_{k} \delta^{i_{k}} \tau^{j_{k}}\right]} \\
\mu=\sum_{i=0}^{4} \sum_{j=0}^{2} \eta_{i j} \delta^{i} \tau^{j} \\
\delta=\frac{\rho}{\rho_{C}} \quad \tau=\frac{T_{C}}{T}
\end{gathered}
$$

\subsection{Physics Models}

Two-phase flow is usually model as an isothermal and immiscible mixture, with incompressible phases. The incompressibility of the gas phase was assumed based on the fact that it is a subsonic flow, with a Mach number of less than 0.3. The Mach number is a ratio between the velocity of the phase and the speed of sound through the fluid at its operating conditions [19].The Volume of Fluid method was used to simulate the flow, using a Surface Tension model to represent the interface between the two fluid phases. As it was a turbulent flow, the k- $\omega$ SST model was used to take the turbulence into account. It must be noted that the phases will be described using the Eulerian method, in which the variation of the flow properties are described at fixed locations as a function of time.

The VOF model assumes that all immiscible fluid phases share the same pressure and velocity fields. As such, the same momentum, mass and energy governing equations used for a single-phase fluid are used to model the two-phase system. The physical and transport properties that are used in these equations are calculated from the volume averages of the properties of the actual phases. The method then solves the equations of momentum and continuity for an equivalent fluid with the averaged properties. It must be noted that this method uses the segregated flow model, which means that it solves each equation separately, which reduces computational time significantly [20].

$$
\frac{d}{d t} \int_{V} \rho \mathrm{dV}+\int_{A} \rho\left(\mathbf{v}-\mathbf{v}_{\mathrm{g}}\right) d \mathbf{a}=\int_{V} S_{u} d V
$$




$$
\frac{d}{d t} \int_{V} \rho \mathbf{v d V}+\int_{A} \rho \mathbf{v} \times\left(\mathbf{v}-\mathbf{v}_{\mathrm{g}}\right) d \mathbf{a}=-\int_{A} p \mathbf{I} \cdot d \mathbf{a}+\int_{A} \mathbf{T} \cdot d \mathbf{a}+\int_{V}\left(\mathbf{f}_{\mathrm{r}}+\mathbf{f}_{\mathrm{g}}+\mathbf{f}_{\mathrm{p}}+\mathbf{f}_{\mathrm{u}}+\mathbf{f}_{\omega}+\mathbf{f}_{\mathrm{L}}\right) d V
$$

The terms on the left side of Eqs. (4) - (5) refer to the transient and convective transport terms. For Eq. (4), the right hand term represents the user-defined source term. For Eq. (5)(4), the right side of the equation has the pressure $(p)$ gradient term, the viscous flux term, that depends on the viscous stress tensor $\mathbf{T}$, and the sum of the body forces, which can be caused by: rotation, gravity, porous media, external forces, vorticity and electromagnetic fields, respectively. For both equations, $\mathbf{v}$ is the velocity vector and $\mathbf{v}_{\mathbf{g}}$ is the velocity of the grid [20].

Along the interface, the immiscibility of the fluids generate a tensile tangential force known as surface tension force. This force is modelled by the VOF method using the Continuum Surface Method (CSF) developed by Brackbill [21]. It must be noted that certain simplifications are usually made in the model, such as that the contact angle between the phases is determined and that the surface tension coefficient $\sigma$ is constant. With these considerations, the model incorporates a source term that represents a body force in the momentum equation shown in Eq. (5). This method can be complemented with additional models, such as the Interface Momentum Dissipation model, which may help reduce unphysical fluid motions, known as parasitic currents that arise at the interface due to discretization errors caused by the solution discontinuities at the free surface between the phases [20].

Given the fact that the flow is not laminar, due to the gas velocity and its low viscosity, it is important to choose an appropriate turbulence model. The one selected is the k- $\omega$ model, which is based on a two-equation system, which is shown in Eqs. (6) - (7), that are solved for the turbulent kinetic energy, k, and the variable $\omega$, which is defined as the dissipation rate per unit turbulent kinetic energy . This model has the advantage, over the alternate model k- $\epsilon$, that it can be applied on the viscous regions of the flow, e.g. the boundary layer near the pipe walls, without further modifications [20]. It is also more computationally effective than the RST model, which requires the solution of seven equation, in comparison with the two that the other models require. The model does present the problem, however, that the boundary layer computation is very sensitive to the inlet boundary conditions. A variation of the model was implemented then, the SST (Shear-Stress Transport) k- $\omega$, which does not have this shortcoming [22].

$$
\begin{aligned}
& \frac{d}{d t} \int_{V} \rho k d V+\int_{A} \rho k\left(\mathbf{v}-\mathbf{v}_{\mathrm{g}}\right) \cdot d \mathbf{a}=\int_{A}\left(\mu+\sigma_{k} \mu_{t}\right) \nabla k \cdot d \boldsymbol{a}+\int_{V}\left(\gamma_{e f f} G_{k}-\gamma^{\prime} \rho \beta^{*} f_{\beta^{*}}\left(\omega k-\omega_{0} k_{0}\right)+S_{k}\right) d V \\
& \frac{d}{d t} \int_{V} \rho \omega d V+\int_{A} \rho \omega\left(\mathbf{v}-\mathbf{v}_{\mathrm{g}}\right) \cdot d \mathbf{a}=\int_{A}\left(\mu+\sigma_{\omega} \mu_{t}\right) \nabla \omega \cdot d \mathbf{a}+\int_{V}\left(G_{\omega}-\rho \beta f_{\beta}\left(\omega^{2}-\omega_{0}^{2}\right)+D_{\omega}+S_{\omega}\right) d V
\end{aligned}
$$

Where $G_{\omega}$ represents the specific rate of dissipation production. $G_{k}$ is the turbulent production. $\sigma_{k}$ and $\sigma_{\omega}$ are inverse turbulent Schmidt numbers. $\mu_{t}$ is the turbulent viscosity. $S_{\omega}$ and $S_{k}$ are user-specified source terms. Finally, $\mathbf{v}$ is the velocity vector and $\mathbf{v}_{\mathbf{g}}$ is the velocity of the grid. The remaining terms refer to parameters specific to the equations [20].

\section{Results and Discussion}

Three different test were performed in order to assess the accuracy and precision of the simulated flow conditions. First, a mesh independence test, which was carried using two different mesh types: orthogonal and polyhedral. Then, an analysis on three different turbulence models was performed. Thirdly, a validation with experimental was done. Finally, based on the previous results, an analysis on the effect of the interface model on the simulation results was started. This analysis is currently still in process.

\subsection{Mesh Independence Test}

In a mesh independence test, several simulations of the same system, with equal geometry, conditions and physics models, are run with different cell numbers. All simulations were compared against the experimental value obtained for the same operating conditions, using the liquid holdup as criterion. As it can be seen on Fig. 3 (a), the fineness of the mesh has

little to no effect on the results of the polyhedral mesh. On the other hand, the orthogonal mesh simulations have more 
dispersed results, so the effect of the spatial discretization is more significant. The result also depends on whether the axial or the radial divisions are manipulated. As it can be seen on Fig. 3 (b), the number of axial divisions appears to have more effect on the simulation results than the radial divisions, especially as the number of axial divisions increases.

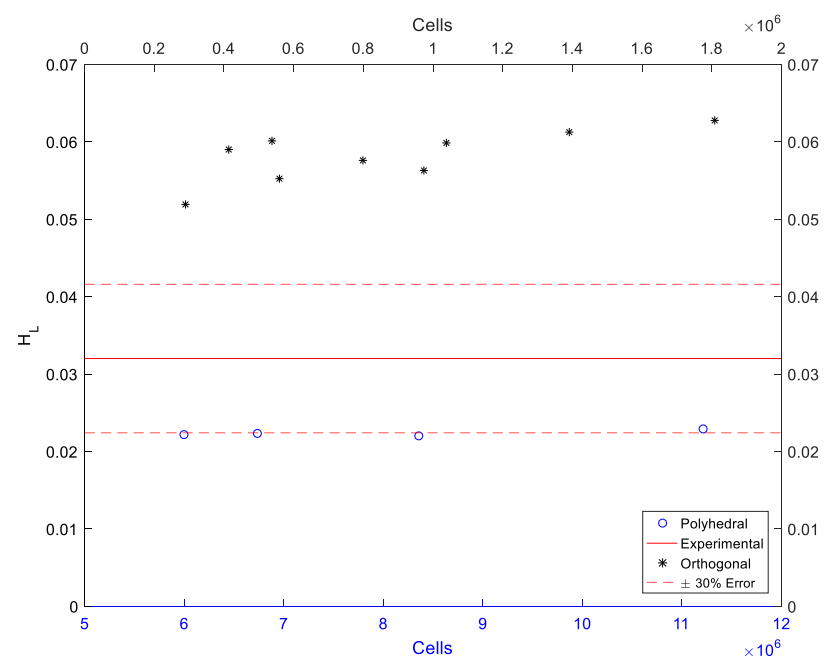

(a)

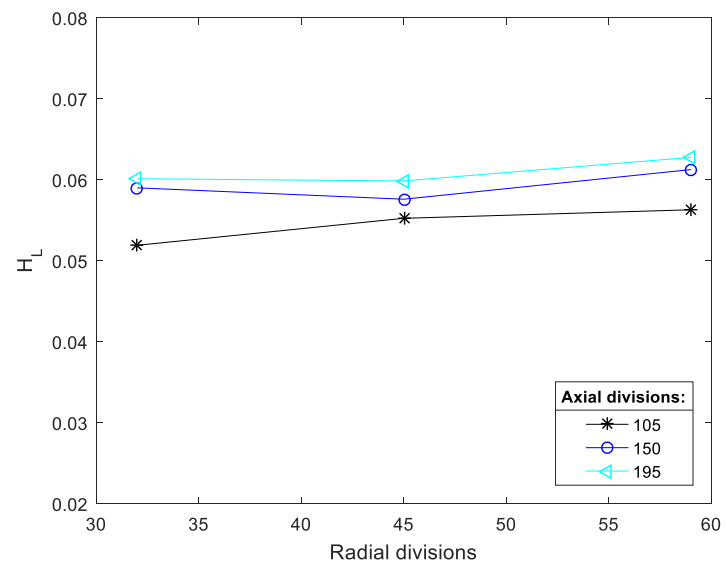

(b)

Fig. 3: Effect of spatial discretization on the liquid holdup for an air - water mixture on a $0.15-\mathrm{m}$ pipe, with a superficial gas velocity of

$10 \mathrm{~m} / \mathrm{s}$ and a liquid velocity of $0.02 \mathrm{~m} / \mathrm{s}$. (a) The effect of the number of cells on the holdup obtained with two different types of meshes: polyhedral and orthogonal. The polyhedral mesh is plotted on the bottom axis, while the orthogonal one is plotted on the top axis. The experimental value is shown as a red line as reference. (b) The effect of axial and radial divisions on the holdup obtained with the orthogonal meshes.

In comparison with the experimental value, it can be seen that the polyhedral mesh has more accurate values, which have in average a $30 \%$ error in relation to the experimental value. The orthogonal mesh, besides having more dispersion, seems to have a larger error in general, of around $80 \%$. The values also do not seem to be converging to a specific value, though that may also be caused by selecting far too coarse meshes for the test. Although the orthogonal mesh has the advantage of requiring fewer cells, which results in lesser computational time, the error in the results is significantly larger than the one from the polyhedral mesh. The orthogonal mesh tends to overpredict the holdup, which may be caused by its larger and elongated cells, compared to the more regular and smaller cells of the polyhedral mesh [13]. This elongated cells may cause the liquid phase to apparently occupy more space that it actually does, as all of the cell will have the same liquid fraction value, which may be more than it should be. Since the liquid phase is so small, this discretization error caused could have a significant effect on the results. Because of this, the polyhedral mesh was selected.

\subsection{Turbulence Model Comparison}

An analysis of the turbulence model was then performed, taking into account three possible models: $\mathrm{k}-\omega, \mathrm{k}-\epsilon$ and RST. The results are shown on Fig. 4. Both the liquid holdup obtained and the computational time required were taken into account for the analysis. It can be seen that all models presented similar values, though the RST model predicted a lower holdup than the rest. All models though tended to underpredict the holdup, when compared to the experimental value. As for the computational time, both $\mathrm{k}-\omega$ and $\mathrm{k}-\epsilon$ models required a similar time for the simulation, probably due to the fact that both consist of a set of two equations. The RST model, nonetheless, required around $150 \%$ more time to run than the other two models, and its results were not more accurate. This large increase in the computational time is most likely due to the fact that it consists of a set of seven equations, six for the Reynolds stresses tensor and one equation for the isotropic turbulent dissipation $\epsilon$. Based on this result, the RST model was discarded as a possible turbulence model. AS for the $\mathrm{k}-\epsilon$, the model had the problem that, unlike the $\mathrm{k}-\omega$ model, it had the equation to be modified when applied in viscous 
regions, i.e. near wall boundaries, which may increase numerical error and make the model less robust. The $\mathrm{k}-\omega$ model was then confirmed as the most appropriate turbulence model to simulate low-liquid level flow [20].

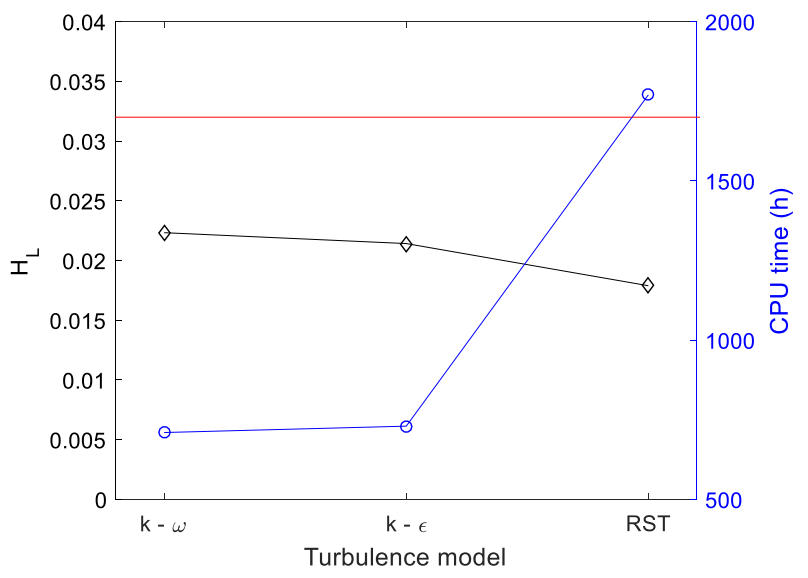

Fig. 4: Effect on the liquid holdup of the turbulence model evaluated for the simulations for an air - water mixture on a 0.15 -m pipe, with a superficial gas velocity of $10 \mathrm{~m} / \mathrm{s}$ and a liquid velocity of $0.02 \mathrm{~m} / \mathrm{s}$ : the $\mathrm{k}-\omega$ model, the $\mathrm{k}-\epsilon$ model and the RST model. The CPU time used can be seen plotted on the right side axis. The experimental value for the holdup is shown as a red line for reference.

\subsection{Experimental Validation}

The physics model was then compared to the experimental data obtained by Duc [10], who used an Isopar-L oil and nitrogen mixture. The liquid holdup was again chosen as the comparison criterion. For the experimental value, the confidence interval was plotted, to see whether the simulation results were significantly different or not. This comparison can be seen on Fig. 5. It can be seen that that the simulation model tends to consistently underpredict the holdup in all cases, as it had been noticed in previous tests. Though the values do not seem to be too far from the confidence interval limit, they are still are statistically different, with an average error of around 50\%. The increase in the error with respect to the previous tests may be due to the change in the fluid properties used for this validation, as the oil is significantly more viscous than water and the nitrogen was significantly denser than air, due to the higher pressure. The difference between the properties can be seen in Table 1. The fact that the model underpredicts the value may be a sign that the forces generated at the interface between the gas and the liquid may had not been correctly modelled, generating parasitic currents that increase the velocity of the liquid phase, which would result in a lower liquid holdup than expected [23]. Because of this, an analysis on the interface models was started.

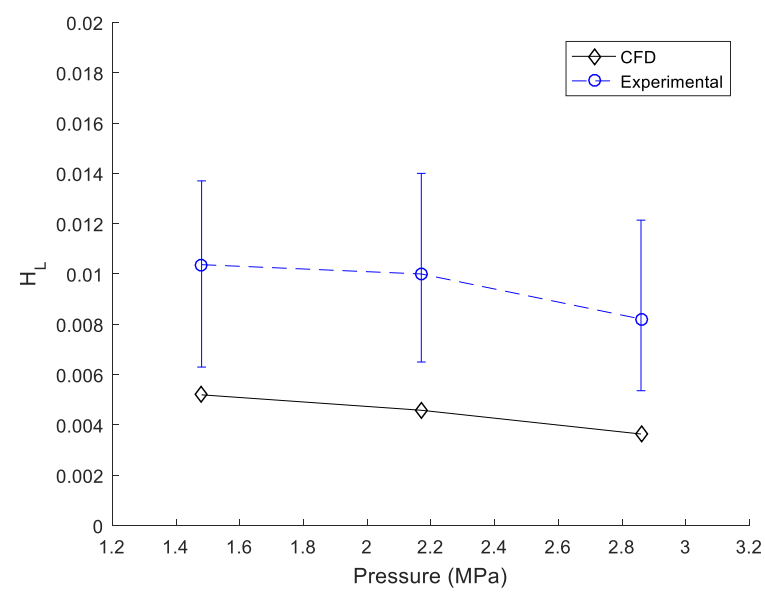

Fig. 5: Experimental comparison with Duc's [10] data, for an Isopar-L oil - nitrogen mixture on a 0.15-m pipe, with a superficial gas velocity of $10 \mathrm{~m} / \mathrm{s}$ and a liquid velocity of $0.02 \mathrm{~m} / \mathrm{s}$ and different operating pressures. The experimental data is presented along with the confidence interval for each measurement. 
Despite the difference between the experimental and the computational values, it can be noticed that both have the same behavior with regard to the operating pressure. As expected, the increase in the gas density and viscosity, which is one of the main effects of the increase in pressure, generates a larger drag force which results in a larger liquid velocity and a smaller hold up. Due to the wide dispersion of the experimental data, however, which resulted in a wide confidence interval, the holdups cannot be determined to be significantly different. The trend in their behaviour can be nonetheless noticed. For the experimental values, the increase of almost $1.4 \mathrm{MPa}$ in the pressure resulted in a decrease of $21 \%$ in the liquid holdup. As for the CFD values, the decrease was of around 30\%, so the change was similar between both types of studies.

\subsection{Interface Model Analysis}

As it was mentioned before, an analysis was done on the way the interface was modelled, using the air - water mixture used for the turbulence model analysis. Two factors were analysed, the angle factor, which affects the discretization of the volume phase fraction and the tracking of the interface, and the inclusion of the Interface Momentum Dissipation model. Though the simulations are still currently running, a preliminary graphical analysis can be done on the difference in the flow behaviour for the various model configuration used, at a simulation time of $0.25 \mathrm{~s}$, which can be seen in Fig. 6 . The first two configurations differ solely on the value of the angle factor, 0.05 and 0.2 , respectively, but a large difference can be seen on how the liquid phase is behaving. The liquid phase in the first case seems to flow at a higher velocity and it appears to be issues at the modelling near the wall, since some air appears to be trapped under the liquid phase, which does not happen with the other configurations. The second configuration seems to have a lower liquid velocity than the first, and, of the three configurations, it appears to have the largest liquid film thickness.

(a)

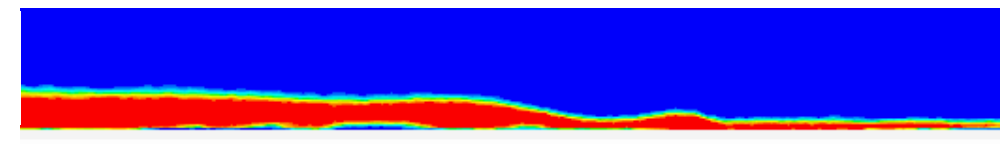

(b)

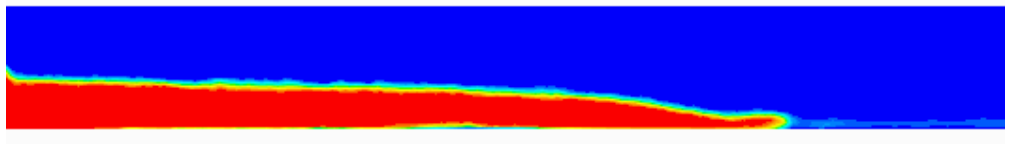

(c)

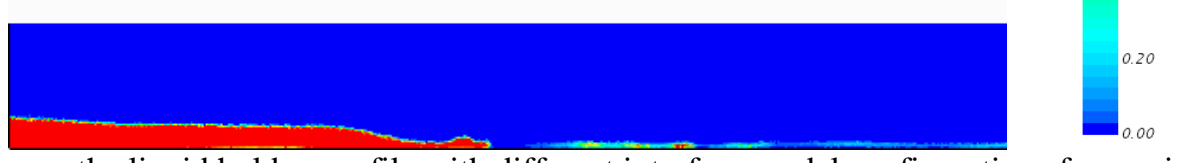

Fig. 6: Comparison between the liquid holdup profile with different interface model configurations for an air-water mixture for a 0.25-s simulation on a $0.15-\mathrm{m}$ pipe, with a superficial gas velocity of $10 \mathrm{~m} / \mathrm{s}$ and a liquid velocity of $0.02 \mathrm{~m} / \mathrm{s}$. The liquid phase is represented as red, and the gas phase, as blue. (a) CSF model with a 0.05 angle factor (b) CSF model with a 0.2 angle factor (c) CSF model with a 0.2 angle factor and the Interface Momentum Dissipation model.

The third configuration, besides having a 0.2 angle factor, also includes the Interface Momentum Dissipation (IMD) model. The liquid phase seems to have the lowest velocity out of the three configurations done, and it seems to have a sharper interface. Though the simulation have not yet reached enough simulation time for a conclusion to be made, this lower phase velocity could result in a higher liquid holdup, and therefore a reduction in the error in comparison to the experimental results. It should be noted, however, that the addition of the IMD model increases the computational cost of the simulation, for the simulation with this configuration runs at barely a third of the speed of the other two configurations [20].

\section{Conclusions}

Low liquid level flow in medium-sized pipes can be simulated in CFD with certain accuracy using the VOF model to represent the multiphase system. However, as the liquid phase is so small and the gas velocity is so large, great care must be taken when selecting the mesh and physics models that represent the pipe system. A polyhedral mesh is more likely to accurately represent the behaviour of the liquid phase, since it cells are smaller and more regular. An orthogonal mesh, due 
to its elongated cells, may overpredict the liquid holdup of the multiphase flow. At the range of cell numbers analysed, the spatial discretization did not seem to affect the simulation results for the polyhedral mesh, but the orthogonal mesh still did not seem to converge to a particular liquid holdup. For an orthogonal mesh, it should be noted that the number of axial divisions tend to have a larger effect on the simulation results than the radial division across the pipe cross-section.

The physics model of the simulations still have to be adjusted to better represent the physical system, as the simulations tend to consistently underpredict the liquid holdup, when compared to experimental results. With respect to the turbulence model, the $\mathrm{k}-\omega$ model seems to be the more appropriate one because of its relatively lower error and low computational cost. The analysis of the interface models must still be completed, as it must be evaluated whether the IMD model has a significant impact on the simulation results, and if the effect compensates the higher computation cost the model requires. Nonetheless, the simulations do predict a correct behaviour with regard to the operating pressure, since the liquid holdup decreased, as the gas density and viscosity increased.

\section{References}

[1] W. Meng et al., "Experimental Study of Low-Liquid-Loading Gas-Liquid Flow in Near-Horizontal Pipes," no. November, 2001.

[2] O. Shoham, "Mechanistic Modeling of gas liquid two phase flow in pipes," pp. 240-250, 2005.

[3] TUV Nel, "Introduction To Wet-Gas Flow Metering," Natl. Meas. Syst., 2010.

[4] A. Hall, D. Griffin, and R. Steven, "Good Practice Guide: An introduction to wet-gas flow metering," NEL. [Online]. Available: http://www.tuvnel.com/_x90lbm/An_Introduction_to_Wet-Gas_Flow_Metering.pdf

[5] S. Corneliussen et al., Handbook of Multiphase Flow Metering, vol. 55, no. 5, 2011.

[6] A. Banafi, M. R. Talaei, and M. J. Ghafoori, "A comprehensive comparison of the performance of several popular models to predict pressure drop in stratified gas-liquid flow with low liquid loading," J. Nat. Gas Sci. Eng., vol. 21, pp. 433-441, 2014.

[7] H. Karami, C. F. Torres, M. Parsi, E. Pereyra, and C. Sarica, "CFD simulations of low liquid loading multiphase flow in horizontal pipelines," Am. Soc. Mech. Eng. Fluids Eng. Div. FEDSM, vol. 2, no. 1984, pp. 1-8, 2014.

[8] S. Badie, C. P. Hale, C. J. Lawrence, and G. F. Hewitt, "Pressure gradient and holdup in horizontal two-phase gasliquid flows with low liquid loading," Int. J. Multiph. Flow, vol. 26, no. 9, pp. 1525-1543, 2000.

[9] L. F. Ayala and M. A. Adewumi, "Low-Liquid Loading Multiphase Flow in Natural Gas Pipelines," J. Energy Resour. Technol., vol. 125, no. 4, p. 284, 2003.

[10] D. H. Vuong, "Pressure effects on two-phase oil-gas low-liquid-loading flow in horizontal pipes," Ph.D. Thesis, University of Tulsa, 2016.

[11] H. Dong, H. . Zhang, and C. Sarica, "Experimental study of low concentration sand transport in low liquid loading water-air flow in horizontal pipes," Multiph. Prod. Technol., no. 4, pp. 17-27, 2014.

[12] S. Ghorai and K. D. P. Nigam, "CFD modeling of flow profiles and interfacial phenomena in two-phase flow in pipes," Chem. Eng. Process. Process Intensif., vol. 45, no. 1, pp. 55-65, 2006.

[13] V. Hernandez-Perez, M. Abdulkadir, and B. J. Azzopardi, "Grid Generation Issues in the CFD Modelling of TwoPhase Flow in a Pipe," J. Comput. Multiph. Flows, vol. 3, no. 1, pp. 13-26, 2011.

[14] H. Karami, C. F. Torres, M. Parsi, E. Pereyra, and C. Sarica, "CFD Simulations of Low Liquid Loading Multiphase Flow in Horizontal Pipelines," Fora Cavitation Multiph. Flow; Fluid Meas. Instrumentation; Microfluid. Multiph. Flows Work Progress; Fluid-Particle Interact. Turbul., vol. 2, p. V002T06A011, 2014.

[15] E. Schleicher et al., "Refined reconstruction of liquid-gas interface structures for stratified two-phase flow using wire-mesh sensor," Flow Meas. Instrum., vol. 46, pp. 230-239, 2015.

[16] US Climate Data, "Climate Tulsa - Oklahoma," 2016. [Online]. Available: https://www.usclimatedata.com/climate/tulsa/oklahoma/united-states/usok0537

[17] R. Span, E. W. Lemmon, R. T. Jacobsen, W. Wagner, and A. Yokozeki, "A reference equation of state for the thermodynamic properties of nitrogen for temperatures from 63.151 to $1000 \mathrm{~K}$ and pressures to $2200 \mathrm{MPa}$," J. Phys. Chem. Ref. Data, vol. 29, no. 6, pp. 1361-1433, 2000.

[18] D. Seibt, E. Vogel, E. Bich, D. Buttig, and E. Hassel, "Viscosity measurements on nitrogen," J. Chem. Eng. Data, vol. 51, no. 2, pp. 526-533, 2006.

[19] Glenn Research Center, "Mach Number," NASA. [Online]. Available: https://www.grc.nasa.gov/www/k- 
12/airplane/machrole.html\#

[20] Siemens, "STAR CCM+ Documentation," NY, 2016.

[21] J. U. Brackbill, D. B. Kothe, and C. Zemach, "A continuum method for modeling surface tension," J. Comput. Phys., vol. 100, no. 2, pp. 335-354, 1992.

[22] F. R. Menter, "Improved Two-Equation k - w Turbulence Models for Aerodynamic Flows," Moffett Field, 1992.

[23] D. J. E. Harvie, M. R. Davidson, and M. Rudman, "An analysis of parasitic current generation in Volume of Fluid simulations," Appl. Math. Model., vol. 30, no. 10, pp. 1056-1066, 2006. 